\title{
Assisting in Auditing of Buffer Overflow Vulnerabilities via Machine Learning
}

\author{
Qingkun Meng, Chao Feng, Bin Zhang, and Chaojing Tang \\ School of Electronic Science and Engineering, National University of Defense Technology (NUDT), Changsha, Hunan, China \\ Correspondence should be addressed to Chao Feng; chaofeng@nudt.edu.cn
}

Received 1 July 2017; Revised 10 October 2017; Accepted 27 November 2017; Published 21 December 2017

Academic Editor: Nazrul Islam

Copyright (C) 2017 Qingkun Meng et al. This is an open access article distributed under the Creative Commons Attribution License, which permits unrestricted use, distribution, and reproduction in any medium, provided the original work is properly cited.

\begin{abstract}
Buffer overflow vulnerability is a kind of consequence in which programmers' intentions are not implemented correctly. In this paper, a static analysis method based on machine learning is proposed to assist in auditing buffer overflow vulnerabilities. First, an extended code property graph is constructed from the source code to extract seven kinds of static attributes, which are used to describe buffer properties. After embedding these attributes into a vector space, five frequently used machine learning algorithms are employed to classify the functions into suspicious vulnerable functions and secure ones. The five classifiers reached an average recall of $83.5 \%$, average true negative rate of $85.9 \%$, a best recall of $96.6 \%$, and a best true negative rate of $91.4 \%$. Due to the imbalance of the training samples, the average precision of the classifiers is $68.9 \%$ and the average $F_{1}$ score is $75.2 \%$. When the classifiers were applied to a new program, our method could reduce the false positive to $1 / 12$ compared to Flawfinder.
\end{abstract}

\section{Introduction}

Buffer overflow occurs when the bytes of data used exceed the prepared allocated boundary on either the stack or the heap. It has been one of the most popular exploitable vulnerabilities since the 1980s. Its hazard to the target victim system ranges from denial of service to executing arbitrary code in administrator permission. Even though this type of vulnerability is not fresh anymore, hundreds of buffer overflow vulnerabilities are still reported every year.

From the perspective of source code, buffer write operations such as array write and memory manipulation provided by programming language like $\mathrm{C} / \mathrm{C}++$ are the main causes of buffer overflow. If not handled properly, even bounded functions like strncpy lead to overflow. Generally, the occurrence of buffer overflow relies on three main characteristics: userinput data controlling the buffer, no validation statement that enforces the use of data inside a safe scope, and the complexity of buffer operations causing the programmer to fail to add proper validation.

Primary methods of automatically detecting buffer overflow fall into two types: static analysis and dynamic testcase generation. Static analysis leverages a pattern to find vulnerabilities, whereas dynamic test-case generation tries to uncover the unexpected behaviors by executing the program with generated test cases. Some open-source static analysis tools can generate too many false positives, which cannot be completely reviewed [1]. Dynamic test generation generally involves fuzzing and symbolic execution. To detect vulnerabilities, fuzzing randomly generates a test case to trigger program faults, while symbolic execution collects constraints when walking through program paths and employs a constraint solver to generate related test cases. Fuzzing cannot understand the program thoroughly and comprehensively, while symbolic execution has problems in terms of path explosion, constraint solving, and memory modeling [2]. As a result, the main work of discovering vulnerabilities still relies on code auditors, which requires an enormous amount of manpower.

In this paper, a static method based on machine learning is proposed to narrow down the search scope of auditing buffer overflow vulnerabilities in source code. There are three contributions in this paper. First, we design seven kinds of static code attributes to represent buffer overflow according to the 22 taxonomies of buffer overflow [3]. Second, we append interprocedural sanitization graph (IPSG) and declarationspread-sink graph (DSSG) to code property graph (CPG) [4] 


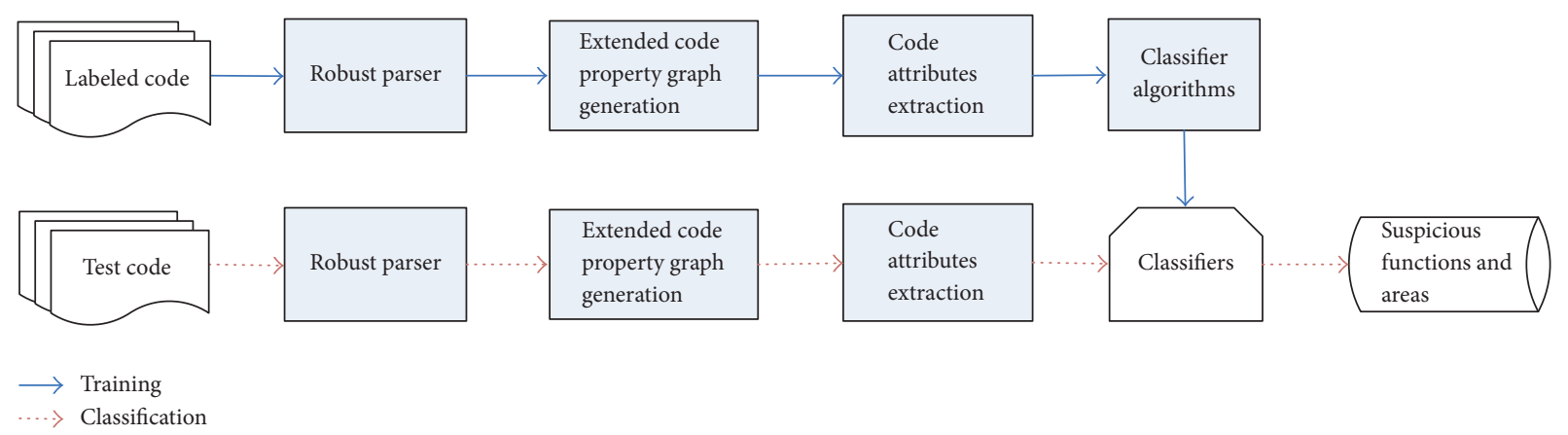

FIGURE 1: Overview of the proposed method.

to form extended property graph (ECPG), which is described in Section 4 in detail, to extract static code attributes in source code. We use ECPG to extract the static code attributes of existing buffer overflow vulnerabilities obtained from common vulnerabilities and exposures (CVE) and map them to vectors. Third, we apply several supervised machine learning algorithms to train classifiers and we apply the classifiers to a new source code base and review only the positive outcomes to reduce manpower in code auditing.

\section{Overview}

The objective of our method is to utilize buffer overflow vulnerabilities that have already been found to assist in auditing vulnerabilities in new software efficiently. The overview of our method is depicted in Figure 1. In this paper, the term "buffer" means the variable that represents a memory region or just a memory region such as swapbuff at line (15) in Algorithm 1, which is also the subject investigated in Section 3. If not otherwise specified, vulnerability means buffer overflow vulnerability.

The purpose of the method illustrated in Figure 1 is to generate suspicious vulnerable functions (SVFs) and related suspicious areas (SAs). SA, in the form of a line number and file name, means the specific area in which buffer overflow may take place, such as line (15) in Algorithm 1. To build a classifier with good performance to distinguish vulnerable buffers from others, the choice of code metrics as the features affects the output significantly. To build a classifier for source code, we extended the static code attributes in [3] by summing up six observable crucial attributes and introducing the sanitization attribute. The details of the static code attribute extraction are described in Section 3.

A robust parser $[5,6]$ is employed to parse source code to Abstract Syntax Tree (AST), which is directly or indirectly used to generate multiple representations. The robust parser allows analysis of code even when a build environment is not configured, which saves a lot of work of compiling source code to machine code. The parser takes advantage of the ANTLR parser generator and $\mathrm{C} / \mathrm{C}++$ grammar definition to extract an AST from individual source files. The AST is used to generate multiple representations consisting of the Control Flow Graph (CFG), Program Dependence Graph (PDG),
TABLE 1: Description of three sink types.

\begin{tabular}{|c|c|c|}
\hline Sink type & Example & $\begin{array}{c}\text { Mapping } \\
\text { value }\end{array}$ \\
\hline Pointer dereference & $* \mathrm{p}++=1$ & 1 \\
\hline Array write & $\mathrm{p}[\mathrm{i}]=1$ & 2 \\
\hline Dangerous function & $\begin{array}{c}\text { strcpy(dst, src), strncpy(dst, } \\
\text { src, n) } \\
\text { strcat(dst, src), strncat(dst, src, } \\
\text { n) } \\
\text { memcpy(dst, src, n), } \\
\text { memmove(dst, src, n) } \\
\text { gets(str), fgets(str, n, fp) }\end{array}$ & 3 \\
\hline
\end{tabular}

interprocedural sanitization graph (IPSG), and declarationspread-sink graph (DSSG). Gathering all the representations, we form an extended code property graph (ECPG). The detailed definitions of IPSG, DSSG, and ECPG are described in Section 4. From ECPG, the code attributes are extracted and then embedded into a vector space. The vectors obtained from labeled code are utilized to train classifiers using some frequently used classifier algorithms. Finally, the vectors obtained from the test code are fed to the classifiers to get SVFs and SAs.

\section{Static Code Attributes and Mapping}

Buffer overflow can be classified into 22 taxonomies [3]. Based on these 22 taxonomies and investigation of many real-world buffer overflow functions from the CVE database, we summarized seven kinds of attributes to represent buffer overflow. All the attributes together are mapped to a multidimensional vector to be fed to a classification algorithm.

3.1. Sink Type. Three sink types are discussed in this subsection, namely, pointer dereference, array write, and dangerous function, as listed in Table 1. If a statement falls into one of the three sink types without proper buffer bound check, a buffer overflow may occur. In $\mathrm{C} / \mathrm{C}++$ language, an array element can be accessed by either pointer dereference or array subscript, but the operations of these two types are different, so we consider them separately. A pointer dereference appears in 


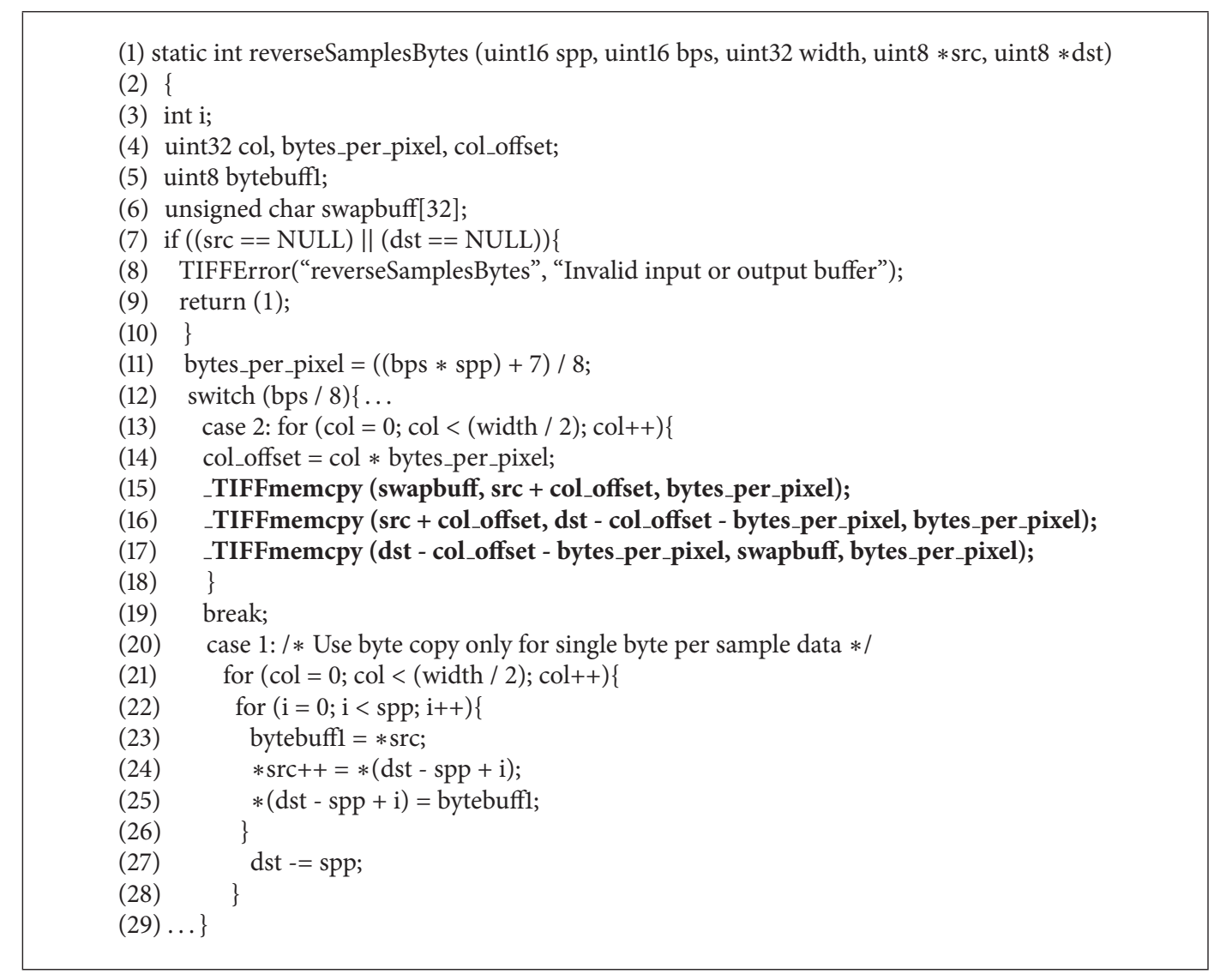

Algorithm 1: Sample code from CVE-2016-9537.

the left part or right part of an assignment statement, like in lines (24) and (25) in Algorithm 1, which are classified as pointer dereference sinks. Dangerous functions are the standard-library or user-defined calls that copy or pad the buffer. Formatted string output also could lead to potential buffer overflow; however, it will not be discussed here. Besides all the standard-library function calls, some userdefined functions have the same effect. For example, CVE2016-9537 [7] in LibTIFF-4.0.6 [8] results from a user-defined function ("-TIFFmemcpy"), shown in lines (15), (16), and (17) in Algorithm 1, which has the same effect as "memcpy" in the standard C library. Thus, user-defined functions that have a similar function name and exactly the same number of parameters are classified into the dangerous function case.

3.2. Memory Location. The memory location attribute represents the location where the sink buffer resides. Five kinds of memory areas that accommodate sink buffer are stack, heap, data segment, BSS segment, and shared memory. These are different from the programming perspective. A stack buffer describes arrays that are defined locally and nonstatically; a heap buffer describes the dynamic allocated memory used to satisfy a large portion of the memory application; a data segment describes static variables or global variables; a BSS segment describes uninitialized global or static variables; and shared memory describes a method of interprocess communication (IPC). In this paper, only the stack, heap, and data segment are considered. We map the memory location to a three-dimensional vector as (stack, heap, and data segment); if the memory location appears, the related entry is set to 1; otherwise it is 0 . For pointer swapbuff in Algorithm 1, the vector representation is $(1,0,0)$ because it is declared locally.

3.3. Container. Container describes the structure in which the sink buffers are wrapped. Generally, the more complex the container structure is, the more vulnerability-prone the buffer will be. According to the investigation of Zitser et al. [9], 7\% of vulnerable buffers are contained in the Union structure, and according to our research on recent CVE buffer overflow vulnerabilities, nearly $30 \%$ of vulnerabilities have containers, so we consider the container of the buffer as another static code attribute. The container attribute is shown in Table 2. The Union and Struct structures are similar in programming; therefore, we map them to the same value. The Others row represents the more complex structures such as the double Union and Struct structures.

3.4. Index/Address/Length Type. Index type records various accumulated operations of array index. The index type attribute comes from the assumption that the more complex 
TABLE 2: Container attributes.

\begin{tabular}{lcc}
\hline Instance & Container type & Mapping value \\
\hline $\mathrm{p}[256]$ & None & 0 \\
$\mathrm{p}[256][256]$ & Array & 1 \\
struct.p[256] & Struct & 2 \\
union.p[256] & Union & 3 \\
Others & Others & \\
\hline
\end{tabular}

TABLE 3: Index type.

\begin{tabular}{lc}
\hline Operation type & Instance \\
\hline Constant & $\mathrm{p}[256]$ \\
Addition & $\mathrm{p}[\mathrm{i}+8], \mathrm{p}[\mathrm{i}-8],(\mathrm{p}+8)[\mathrm{i}],(\mathrm{p}-8)[\mathrm{i}]$ \\
Multiplication & $\mathrm{p}[\mathrm{i} * 8], \mathrm{p}[\mathrm{i} / 8], \mathrm{p}[\mathrm{i}>>8], \mathrm{p}[\mathrm{i}<<8],(\mathrm{buf}+8 * \mathrm{n})[\mathrm{i}]$ \\
Nonlinear & $\mathrm{p}[\mathrm{i} \% 8], \mathrm{p}[$ pow $(\mathrm{i}, \mathrm{j})], \mathrm{p}[\operatorname{sqrt}(\mathrm{i})]$ \\
Function call & $\mathrm{p}[\mathrm{f}(\mathrm{i})],(\mathrm{p}+\mathrm{f}(\mathrm{n}))[\mathrm{i}],(\operatorname{get}$ Adtres $(\mathrm{n}))[\mathrm{i}]$ \\
Array access & $\mathrm{p}[\mathrm{buf}[\mathrm{i}]],(\mathrm{p}+\mathrm{buf}[\mathrm{n}])[\mathrm{i}],($ buf $[\mathrm{n}])[\mathrm{i}]$ \\
\hline
\end{tabular}

array index operations are, the more error-prone buffer access will be. Array index operations can be divided into six categories, namely, constant, addition, multiplication, nonlinear, function call, and array access. Each category will be mapped to one dimension to construct a six-dimensional vector.

Addition contains addition and subtraction operations. Multiplication contains multiplication, division, bitwise left shift, and bitwise right shift operations. Nonlinear contains modulo and other functions such as pow() and sqrt() operations. Function call describes the situation that a function (except the function call contained in nonlinear) returns a value involved in a buffer index. Array access indicates whether an array content read operation is involved in a buffer index. Index type is described in Table 3. The six operation types contribute, to different extents, to buffer overflow. Despite the difficulty of exploiting vulnerabilities, we find some buffer overflows that are caused by buffer access with a constant index and we denote this type as the constant type. Besides the constant type, each of the others has two different forms, such as $p[i-8]$ and $(p-8)[i]$.

Figure 2 is the schematic depiction of the accumulating index operation. In the case of $p[i]$, we accumulate the operation of $i$ along with the data flow. Finally, the index type of $i$ is converted to a 6 -dimensional vector $(0,1,0,0,1,1)$.

Address and length type attributes, which are described in Tables 4 and 5, respectively, are akin to the index type except for the difference in their sink types. We map index/address/length type into a six-dimensional vector and the corresponding value is increased by one when the related operation is encountered. For pointer dereference sink buffers $s r c$ and $d s t$ at line (24) and line (25) in Algorithm 1, the values of address type are $(0,1,0,0,0,0)$ and $(0,3,0,0,0,0)$, respectively. For dangerous function sink buffers at lines (15), (16), and (17), the values of length type are the same, namely, $(0,1,2,0,0,0)$. Besides, index/address/length type takes buffer aliasing into consideration and the operations of the alias buffer should be accumulated, too.

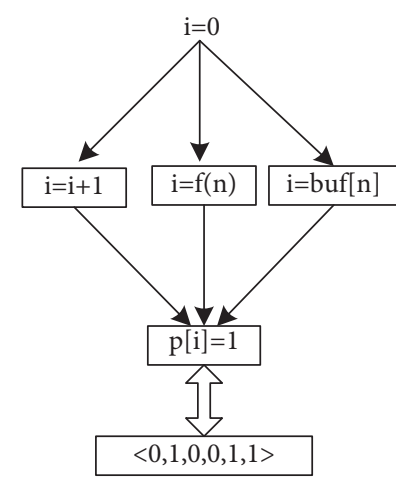

Figure 2: Accumulating the index type of $i$.

TABLE 4: Address type.

\begin{tabular}{lc}
\hline Operation type & Instance \\
\hline Constant & not appliable \\
Addition & $* \mathrm{p}(\mathrm{i}+8), * \mathrm{p}(\mathrm{i}-8)$ \\
Multiplication & $*(\mathrm{p}+\mathrm{i} * 8), * \mathrm{p}(\mathrm{i} / 8), * \mathrm{p}(\mathrm{i}>>8), * \mathrm{p}(\mathrm{i}<<8)$ \\
Nonlinear & $*(\mathrm{p}+\mathrm{i} \% 8), *(\mathrm{p}+$ pow $(\mathrm{i}, \mathrm{j})), *(\mathrm{p}+\operatorname{sqrt}(\mathrm{i}))$ \\
Function call & $*(\mathrm{p}+\mathrm{f}(\mathrm{i})), *(\operatorname{get}$ Address ()$)$ \\
Array access & $*(\mathrm{p}+\operatorname{buf}[\mathrm{i}])$ \\
\hline
\end{tabular}

TABLE 5: Length type.

\begin{tabular}{lc}
\hline Operation type & Instance \\
\hline Constant & memcpy (dest, src, 256) \\
Addition & memcpy (dest, src, i+256) \\
Multiplication & memcpy (dest, src, i $* 8)$ \\
Nonlinear & memcpy (dest, src, i\%8) \\
Function call & memcpy (dest, src, f(n)) \\
Array access & memcpy (dest, src, buf[255]) \\
\hline
\end{tabular}

3.5. Sanitization. Sanitization is the bound check operation of buffers. Even though the existence and correctness of the sanitization cannot be precisely obtained from static analysis, the pattern of bound check can be summarized to estimate them. If statements fall into several modes, we argue that the programmer might have considered sanitization and the more times the modes appear, the higher the possibility that the programmer has added the sanitization. Sanitizations are classified into three types:

(1) Direct sanitization: provided variable $b$ is a sink buffer or sink buffer index; $c$ is a condition statement that has a control flow to $b$ and $b$ is a subexpression of $c$; we will increase the sanitization value by one. The sink buffer swapbuff in Algorithm 2, line (1), and the bound checks on array indexes fall into this scope

(2) Indirect sanitization: given a data flow from variable $a$ to variable $b$ ( $b$ is a sink buffer index or involved in a buffer index expression), if $a$ is involved in a condition statement $c$, we will increase the value of indirect sanitization by one. Figure 3(a) is a code example, 


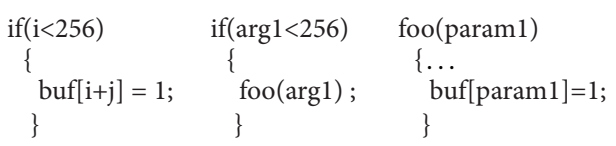

(a)

(b)

FIGURE 3: Code example of indirect sanitization and interprocedural sanitization.

(1) if(bytes_per_pixel > sizeof(swapbuff))

(2) \{ TIFFError("reverseSamplesBytes", "bytes_per_pixel too large”);

(3) return (1);

(4) \}

Algorithm 2: Patch of CVE-2016-9537 in Algorithm 1.

where the expression $i+j$ is the buffer index of buf and $i$ is involved in a condition statement

(3) Interprocedural sanitization: we found that many buffer overflow vulnerabilities are patched by sink function argument sanitization. Therefore, if there is a data flow between arguments and sink buffer index and also the arguments are involved in a condition statement in the superior function, we will increase sanitization value by one. Figure $3(\mathrm{~b})$ is a code example where param 1 is a buffer index and parameter of foo, and $\operatorname{argl}$ is involved in a condition statement in the superior function

Additionally, we add some exceptions, where a condition statement falls into the description of three kinds of sanitizations but does not count as a sanitization. For example, the condition statement at line (7) in Algorithm 1 cannot be regarded as a sanitization, because comparing $s r c$ or $d s t$ against NULL is not for bound checking of buffer $s r c$ and $d s t$. We map the sanitization attribute into a three-dimensional vector. When a type appears, the corresponding value of the sanitization attribute will be increased by one.

3.6. Loop/Condition/Call Depth. Loop/condition/call depth reflects the complexity of the program which leads to program faults. Loop/condition depth describes the maximum hierarchy of the loop/condition statement that wraps the sink statement. Call depth describes the maximum number of function calls from main to the current function, which can be obtained by declaration-spread-sink graph, described in Section 4 in detail. We map these three attributes into a three-dimensional vector and the corresponding value is increased by one when a related situation is encountered. For sink variable src in Algorithm 1, the call chain is main $\rightarrow$ createCroppedImage $\rightarrow$ mirrorImage $\rightarrow$ reverseSamplesBytes, and we consider a switch statement as a condition statement, so the vector is $(2,1,4)$.

3.7. Taint. Taint indicates whether there exists a data flow from source input to sink statement. Taint can be classified into five types, namely, command line, environment variable, file input, network transmission, and argument inflow. The former four types are usually characterized by standard-library function calls such as scanf, getwd, fscanf, and recvfrom. Argument inflow describes whether there is a data flow between arguments and the sink statements. If a sink buffer falls into any of the taint types, it is attacker-controlled and we assign 1 to the attribute; otherwise, 0 is assigned.

Based on these seven kinds of static code attributes, we can construct an 18-dimensional vector, which is then used to train the classifiers.

\section{Extended Code Property Graph}

We extract static code attributes based on an extended code property graph. The following content introduces the basic theory of property graphs and extended property graphs and then explains the fundamental traversals to get specific static code attributes.

4.1. Property Graph Theory. A property graph [10] can be formally defined as $G=(V, E, \lambda, \mu)$, where $V$ is a set of nodes, $E \subseteq\{V \times V\}$ is a multiset of directed edges, $\lambda: E \rightarrow \Sigma$ is a label function, $\Sigma$ is a set of edge labels, and $\mu:(V \cup E) \times K \rightarrow S$ is a key-value mapping function that maps property keys of nodes and edges to their values, where $K$ is a key set and $S$ is the set of property values. What makes a property graph more expressive is that it contains multiple key-value maps on every node and edge.

Graph traversal is a procedure to search for a proper node or edge with certain preconditions. The fundamental traversals are to search for the value of a node or edge given the key and the in and out edges of nodes, which are described in the equations below. Through traversal composition, complex traversal can be performed to explore an arbitrary node or edge.

$$
\begin{aligned}
& \varepsilon: \mathrm{P}(V \cup E) \times K \longrightarrow \mathrm{P}(S), \\
& e_{\text {in }}: \mathrm{P}(V) \longrightarrow \mathrm{P}(E), \\
& e_{\text {out }}: \mathrm{P}(V) \longrightarrow \mathrm{P}(E) \text {, }
\end{aligned}
$$




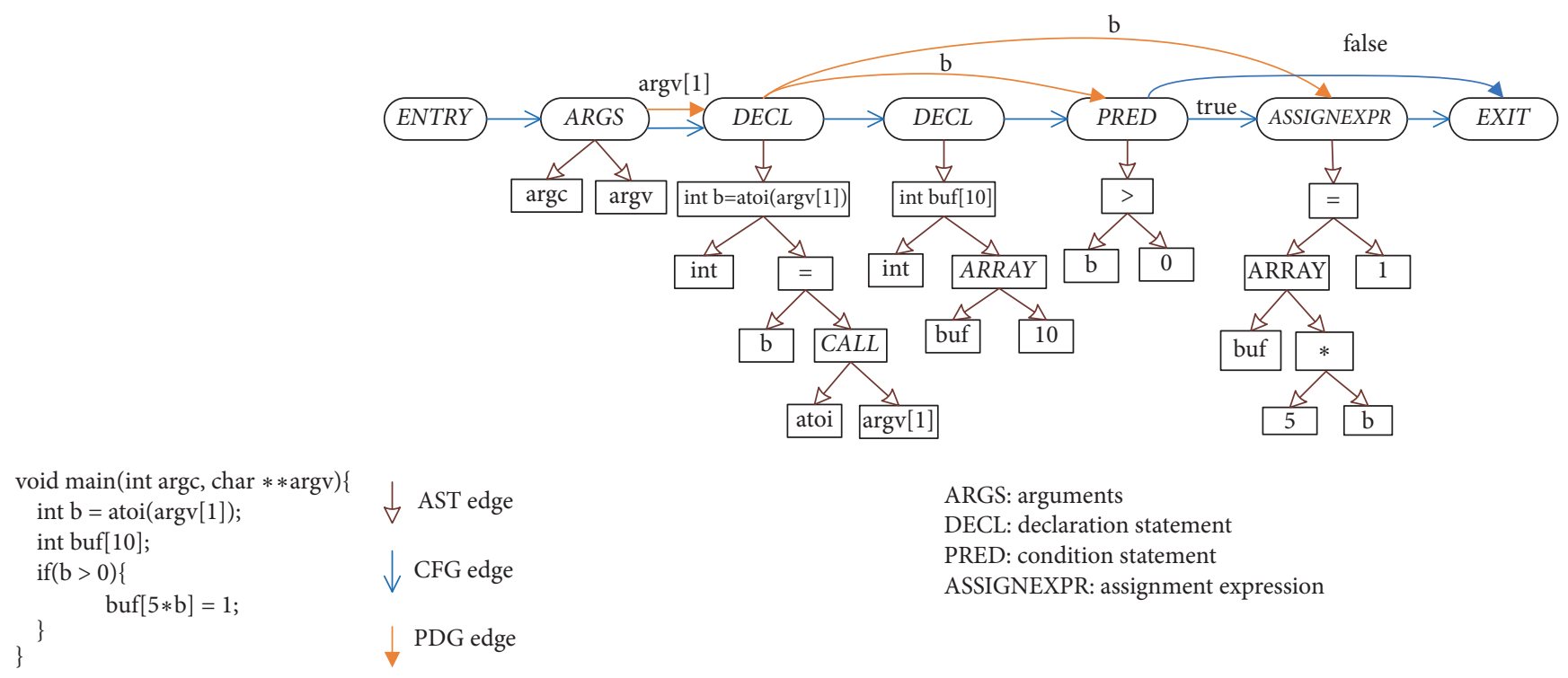

(a)

(b)

FIGURE 4: Schematic representation of CPG.

$$
\begin{gathered}
v_{\text {in }}: \mathrm{P}(E) \longrightarrow \mathrm{P}(V), \\
v_{\text {out }}: \mathrm{P}(E) \longrightarrow \mathrm{P}(V) .
\end{gathered}
$$

4.2. Extended Code Property Graph. CPG (code property graph) [4] is a joint representation of AST (Abstract Syntax Tree), CFG (Control Flow Graph), and PDG (Program Dependence Graph). Figure 4 is a schematic representation of CPG, where Figure 4(a) is the related exemplary code. All nodes are linked by different edges and we can search any edges and nodes through the composition of traversals on edges and nodes. There are three kinds of edges in Figure 4, namely, the AST edge, CFG edge, and PDG edge. Every operator and operand from the source code can be accessed through traversal by AST edge, so the sink type and container attribute can be easily obtained. Memory location and taint require AST and PDG. Condition/loop depth requires both AST and CFG. Direct and indirect sanitization and index/address/length type need all three types of graphs.

However, code property graphs cannot handle interprocedural sanitization and call depth, because they need interprocedural data flow and control flow. To solve this problem, we append the interprocedural sanitization graph (IPSG) and declaration-spread-sink graph (DSSG) to CPG to form the extended code property graph (ECPG).

IPSG is a property graph: $G=\left(V_{\mathrm{A}}, E_{\mathrm{IP}}, \lambda_{\mathrm{IP}}, \mu_{\mathrm{IP}}\right)$. In the formula, $V_{\mathrm{A}}$ is the AST node set; $E_{\mathrm{IP}}$ links the predicate node to the sink statement of the invocation function, where the predicate actually sanitizes the invocation and the parameters of the invocation function data control the sink statement; $\lambda_{\text {IP }}$ is the labeling function, $\lambda_{\mathrm{IP}}: E_{\mathrm{IP}} \rightarrow \Sigma_{\mathrm{IP}}$, where $\Sigma_{\mathrm{IP}}=\{\mathrm{IC}, \mathrm{ID}\}$ and IC corresponds to interprocedural control dependency. We assign a property symbol to indicate ID and a property condition to indicate IC. Figure 6(a) is the representation of

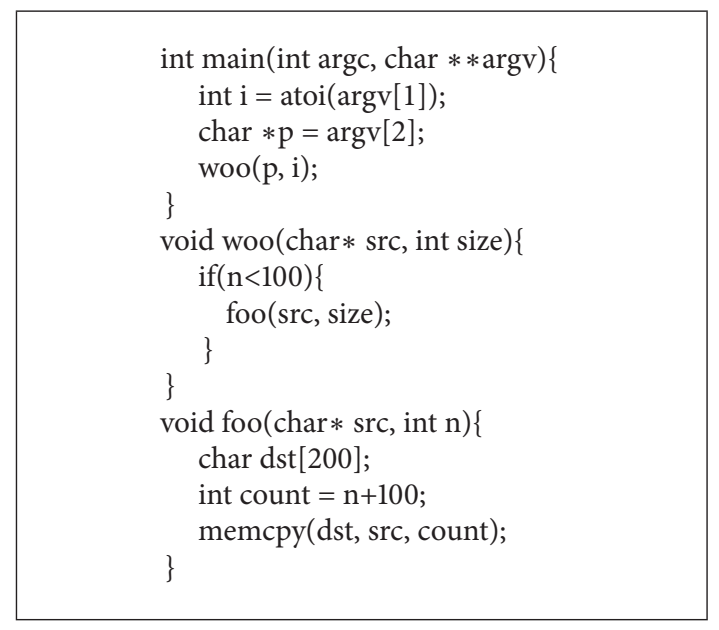

Algorithm 3: Example code for explanation of IPSG and DSSG.

IPSG of code from Algorithm 3 and Figure 5 is the CPG of the code from Algorithm 3, which delete the detailed AST nodes and edges. In order to generate IPSG edge, the predicate if (size < 100) must control and sanitize foo. Also, the arguments of memcpy src and count must be data-dependent on the parameters of foo, src, and $n$.

DSSG is a property graph: $G=\left(V_{\mathrm{A}}, E_{\mathrm{DS}}, \lambda_{\mathrm{DS}}, \mu_{\mathrm{DS}}\right)$. In the formula, $V_{\mathrm{A}}$ is the AST node set; $E_{\mathrm{DS}}$ links the functions $f_{1}$ and $f_{2}$, if $f_{1}$ invokes $f_{2} ; E_{\mathrm{DS}}$ also links sink function node to sink statement; $\lambda_{\mathrm{DS}}$ is the labeling function, $\lambda_{\mathrm{DS}}$ : $E_{\mathrm{DS}} \rightarrow \Sigma_{\mathrm{DS}}$, where $\Sigma_{\mathrm{IP}}=\{\mathrm{DS}\}$. We also assign a property symbol to indicate the symbols transmitted from function node to function node and from function node to sink statement. Figure 6(b) is the representation of DSSG of code from Algorithm 3. DSSG not only helps collect call depth 


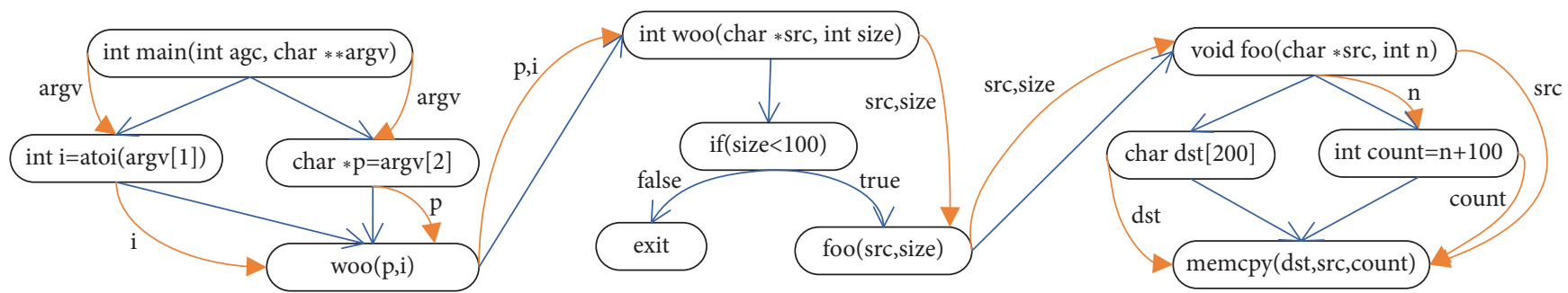

$\rightarrow$ PDG edge

$\rightarrow$ CFG edge

Figure 5: Simplified CPG of code for Algorithm 3.

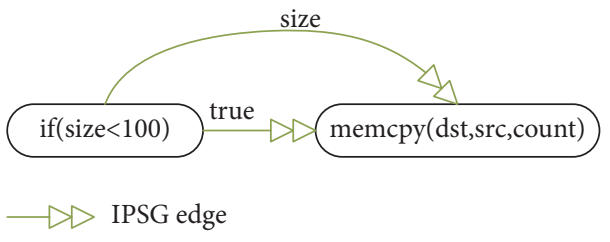

(a)

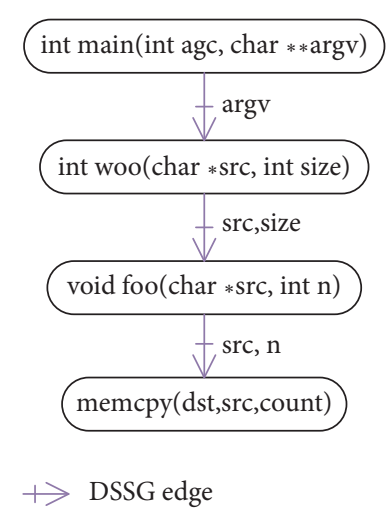

(b)

FIGURE 6: Representations of (a) IPSG and (b) DSSG of code from Algorithm 3.

information but also conveys the original input source of sink statement, which is very helpful to do further analysis.

Combining IPSG, DSSG, and CPG (containing AST, CFG, and PDG), ECPG can be formally represented as $G=$ $(V, E, \lambda, \mu)$, where

$$
\begin{aligned}
& V=V_{\mathrm{A}}, \\
& E=E_{\mathrm{A}} \cup E_{\mathrm{C}} \cup E_{\mathrm{P}} \cup E_{\mathrm{IP}} \cup E_{\mathrm{DS}}, \\
& \lambda=\lambda_{\mathrm{A}} \cup \lambda_{\mathrm{C}} \cup \lambda_{\mathrm{P}} \cup \lambda_{\mathrm{IP}} \cup \lambda_{\mathrm{DS}}, \\
& \mu=\mu_{\mathrm{A}} \cup \mu_{\mathrm{C}} \cup \mu_{\mathrm{P}} \cup \mu_{\mathrm{IP}} \cup \mu_{\mathrm{DS}} .
\end{aligned}
$$

In the formula, $V_{\mathrm{A}}$ is the AST node set. $E_{\mathrm{A}}, E_{\mathrm{C}}, E_{\mathrm{P}}, E_{\mathrm{IP}}$, and $E_{\mathrm{DS}}$ are edges of AST, CFG, PDG, IPSG, and DSSG; $\lambda_{\mathrm{A}}$, $\lambda_{\mathrm{C}}, \lambda_{\mathrm{P}}, \lambda_{\mathrm{IP}}$, and $\lambda_{\mathrm{DS}}$ are label functions; $\mu_{\mathrm{A}}, \mu_{\mathrm{C}}, \mu_{\mathrm{P}}, \mu_{\mathrm{IP}}$, and $\mu_{\mathrm{DS}}$ are key-value mapping functions.

\subsection{Extracting Static Code Attributes Using Traversals. After} building the ECPG, we shall design traversals to collect code attributes. Here we introduce two fundamental traversals $\operatorname{OUT}_{l}^{k, s}(X)$ and $\mathrm{IN}_{l}^{k, s}(X)$, which are formally defined below. Given a node, $\mathrm{OUT}_{l}^{k, s}(X)$ finds the nodes that are reachable through outgoing edge confined by label $l$ and property $k: s$, where $X$ denotes the complete node set of ECPG. Similarly,
$\mathrm{IN}_{l}^{k, s}(X)$ finds the nodes that are reachable through ingoing edge.

$$
\begin{aligned}
& \operatorname{OUT}_{l}^{k, s}(X) \\
& =\bigcup_{v \in X}\{u:(v, u) \in E, \lambda((v, u))=l, u((v, u), k)=s\}, \\
& \mathrm{IN}_{l}^{k, s}(X) \\
& =\bigcup_{u \in X}\{v:(v, u) \in E, \lambda((v, u))=l, u((v, u), k)=s\} .
\end{aligned}
$$

Furthermore, we also introduce TNodes $(X)$ to search the set of AST nodes reachable from the AST root, which can be formulated as follows. In the formula, $v$ is a node from $X$, and Out $_{\mathrm{A}}$ is the representation of the outgoing edge of $v$ used to find its child node $v_{c}$. TNodes $(X)$ indicates that all its AST child nodes can be traversed given any node in $X$.

TNodes $(X)$

$$
=\bigcup_{v \in X}\left(v \cup\left(\bigcup_{v_{c} \in \operatorname{out}_{\mathrm{A}}(\{v\})} \operatorname{TNodes}\left(\left\{v_{c}\right\}\right)\right)\right) .
$$

Upon the three traversals, all the nodes and edges can be explored in ECPG, so the compositional traversals can be designed to extract static code attributes. 
TABLE 6: Confusion matrix.

\begin{tabular}{cccc}
\hline & Total population & \multicolumn{2}{c}{ Predicted class } \\
& $\begin{array}{c}\text { Predicted } \\
\text { positive }\end{array}$ & $\begin{array}{c}\text { Predicted } \\
\text { negative }\end{array}$ \\
\hline \multirow{4}{*}{ Actual class } & Actual positive & $\begin{array}{c}\text { True positive } \\
\text { (TP) }\end{array}$ & $\begin{array}{c}\text { False negative } \\
\text { (FN) }\end{array}$ \\
& Actual negative & False positive & True negative \\
& (FP) & $(\mathrm{TN})$ \\
\hline
\end{tabular}

\section{Experiment}

The experiment was conducted on a PC with Intel(R) Xeon(R) CPU E3-1231 v3 @ 3.40 GHz CPU and 16.0 GB memory, using Ubuntu 14.04.4 LTS. We use scikit-learn to implement the machine learning algorithms [11], which are built on python libraries such as Numpy, SciPy, and matplotlib.

5.1. Evaluation Metrics. In the field of machine learning, the confusion matrix [12] is a general applied metric to assist in understanding the performance of a classifier, as described in Table 6. The matrix describes the mixtures between actual classes and predicted classes, namely, true positive (TP), false negative (FN), false positive (FP), and true negative (TN). TP represents the case when a vulnerable function is classified as positive. FN represents the case when a vulnerable function is classified as negative. FP represents the case when a nonvulnerable function is classified as positive. TN represents the case when a nonvulnerable function is classified as negative. In a normal program, the number of vulnerable functions is much less than that for nonvulnerable functions, which would make the training data and test data unbalanced, so we additionally leverage four other assessment metrics: recall, true negative rate (TNR), precision, and $F_{1}$ score. Recall is also known as true positive rate (TPR) and precision is also known as positive predictive value (PPV). The formula is described as follows. Recall describes the proportion of TPs to all buffer overflows; TNR represents the proportion of TNs to all nonvulnerable functions. Precision represents the proportion of TPs to all predicated positives. $F_{1}$ is a measure of test's accuracy which considers both the precision and recall.

$$
\begin{aligned}
\text { recall } & =\frac{\mathrm{TP}}{(\mathrm{TP}+\mathrm{FN})}, \\
\mathrm{TNR} & =\frac{\mathrm{TN}}{(\mathrm{FP}+\mathrm{TN})}, \\
\text { precision } & =\frac{\mathrm{TP}}{(\mathrm{TP}+\mathrm{FP})}, \\
F_{1} & =2 \times \frac{(\text { recall } \times \text { precision })}{(\text { recall }+ \text { precision })} .
\end{aligned}
$$

5.2. Experiment and Comparison. This section contains evaluations of the five classifiers. As shown in Table 7, we investigated 58 vulnerable functions manually from the official CVE database together with 174 functions that are not vulnerable in recent years. For all the functions, we select a buffer to represent each one to extract attributes. Columns Vul-Num and Not-Vul-Num display vulnerable and nonvulnerable function numbers. Vulnerable functions are labeled 1, while nonvulnerable functions are labeled 0 . The samples originate from eight open-source programs of various versions, which are ffmpeg, HDF5, libtff, mupdf, openssl, qemu, zziplib, and blueZ.

Different classifier algorithms are employed to train classifiers because which algorithm is better is not predicable. To evaluate our static analysis, five well-known supervised machine learning algorithms, $K$-Nearest Neighbors (KNN), Decision Tree (DT), Naive Bayes (NB), AdaBoost, and Support Vector Machines (SVM), are used. 10-Fold crossvalidations are performed on the 232 labeled pieces of data employing the five classifier algorithms. The parameter $k$ used in KNN is 7. The specific DT algorithm we use is C4.5. The number of weak classifiers used in AdaBoost is 20. In SVM, we use the RBF kernel; the parameter $C$ is 10 and $\gamma$ is 0.01 .

5.2.1. Evaluation on Test Suite. The performances of the five classifier algorithms are listed in Table 8 . The average recall is $83.5 \%$, which means that 48 out of 58 vulnerabilities are detected through our method. The average TNR is $87.3 \%$, which means that nearly 152 out of 174 nonvulnerable functions are classified correctly. The average precision is $68.9 \%$ and the average $F_{1}$ is $75.2 \%$, which are not very high because the number of nonvulnerable functions is three times as many as vulnerable functions. In the field of vulnerability detection, finding the largest possible number of vulnerabilities is more important. Therefore, we deem NB the best classifier, since it outperforms the other algorithms to detect 56 vulnerabilities with the highest recall of $96.6 \%$.

5.2.2. Comparison to BOMiner. In [13], a tool, BOMiner, is implemented to predict buffer overflows using machine learning. However, it focuses too much on pointer reference sinks and overfits the result of the classifiers. We try to compare with BOMiner on features selection using the five classifier algorithms. Table 9 shows the performance of BOMiner on our test suite. The maximum number of vulnerabilities correctly classified by BOMiner is 38, which is 4 less than that by our worst classifier KNN and 18 less than that by our best classifier, NB. The highest recall of BOMiner is $65.5 \%$, which is also less than that for all our classifiers. Figure 7(a) shows the comparison of average confusion matrix. On average, our method classifies 14.8 more TPs and 10.4 more TNs than BOMiner. Figure 7(b) shows the comparison of recall, TNR, precision, and $F_{1}$. The average recall is increased by $25.6 \%$, average TNR is increased by $6 \%$, average precision is increased by $17.5 \%$, and the average $F_{1}$ is increased by $21.1 \%$. The main reason why our method outperforms BOMiner is that, in [13], the array write sink is not considered in detail. When it encounters an array write sink, BOMiner has too little information to classify it correctly. 
TABLE 7: CVE list for attribute extraction.

\begin{tabular}{|c|c|c|c|}
\hline Program & CVE-ID & Vul-Num & Not-Vul-Num \\
\hline ffmpeg & $\begin{array}{l}\text { CVE-2016-7562, CVE-2016-6920, } \\
\text { CVE-2016-10192, CVE-2016-10191, } \\
\text { CVE-2016-10190, CVE-2016-8364, } \\
\text { CVE-2014-5271, CVE-2014-3157, } \\
\text { CVE-2014-2263, CVE-2013-0894, } \\
\text { CVE-2013-0868, CVE-2013-0863, } \\
\text { CVE-2012-0947, CVE-2012-0857, } \\
\text { CVE-2012-0856, CVE-2012-0855, } \\
\text { CVE-2012-0848, CVE-2012-0847 }\end{array}$ & 18 & 54 \\
\hline HDF5 & CVE-2016-4333, CVE-2016-4330 & 2 & 6 \\
\hline LibTIFF & $\begin{array}{l}\text { CVE-2017-5225, CVE-2016-9540, } \\
\text { CVE-2016-9537, CVE-2016-9536, } \\
\text { CVE-2016-9535, CVE-2016-9533, } \\
\text { CVE-2016-5652, CVE-2016-5319, } \\
\text { CVE-2016-5318, CVE-2016-5102, } \\
\text { CVE-2016-3991, CVE-2016-3990, } \\
\text { CVE-2016-3632, CVE-2016-3624, } \\
\text { CVE-2015-8784, CVE-2015-8782, } \\
\text { CVE-2013-4244, CVE-2013-4231, }\end{array}$ & 18 & 54 \\
\hline mupdf & $\begin{array}{l}\text { CVE-2017-5869, CVE-2016-6525, } \\
\text { CVE-2014-2013, CVE-2011-0341 }\end{array}$ & 4 & 12 \\
\hline openssl & $\begin{array}{l}\text { CVE-2016-2182, CVE-2015-0235, } \\
\text { CVE-2014-3512 }\end{array}$ & 3 & 9 \\
\hline qemu & $\begin{array}{l}\text { CVE-2016-7170, CVE-2016-5238, } \\
\text { CVE-2016-4439, CVE-2013-4151, } \\
\text { CVE-2013-4150 }\end{array}$ & 5 & 15 \\
\hline zziplib & $\begin{array}{l}\text { CVE-2017-5976, CVE-2017-5975, } \\
\text { CVE-2017-5974, CVE-2017-1614 }\end{array}$ & 4 & 12 \\
\hline BlueZ & $\begin{array}{l}\text { CVE-2016-9917, CVE-2016-9804, } \\
\text { CVE-2016-9803, CVE-2016-9800 }\end{array}$ & 4 & 12 \\
\hline
\end{tabular}

TABle 8: Performances of our five classifier algorithms.

\begin{tabular}{lcccccccc}
\hline & TP & FN & FP & TN & Recall (\%) & TNR (\%) & Precision (\%) & $F_{1}(\%)$ \\
\hline KNN & 42 & 16 & 20 & 154 & 72.4 & 88.5 & 67.7 & 70 \\
DT & 44 & 14 & 31 & 143 & 75.9 & 82.2 & 58.7 & 66.2 \\
NB & 56 & 2 & 27 & 147 & 96.6 & 84.5 & 7.5 & 79.5 \\
AdaBoost & 46 & 12 & 15 & 159 & 79.3 & 91.4 & 77.3 \\
SVM & 54 & 4 & 18 & 156 & 93.1 & 89.7 & 75 \\
\hline
\end{tabular}

TABLE 9: Performance of BOMiner for our test suite.

\begin{tabular}{lcccccccc}
\hline & TP & FN & FP & TN & Recall (\%) & TNR (\%) & Precision $(\%)$ & $F_{1}(\%)$ \\
\hline KNN & 29 & 29 & 21 & 153 & 50 & 87.9 & 58 & 53.7 \\
DT & 31 & 27 & 35 & 139 & 53.4 & 79.9 & 57 & 57.6 \\
NB & 38 & 20 & 46 & 128 & 65.5 & 53.9 & 53.2 \\
AdaBoost & 33 & 25 & 28 & 146 & 56.9 & 81 & 54.1 & 52.9 \\
SVM & 37 & 21 & 33 & 141 & 63.8 & & 55.5 \\
\hline
\end{tabular}

5.2.3. Comparison to Joern. Yamaguchi et al. [4] developed a platform, Joern, which can detect buffer overflows with very few FPs, based on code property graph. As reported in [4], six buffer overflows in drivers directory of linux kernel3.11 source code are detected through specific pattern. However, as we investigated from CVE database, there are 18 buffer overflows and 179 nonvulnerable sink functions in drivers directory of linux kernel3.11. We apply the proposed method to the code base and the result is compared to Joern in Table 10. Using our proposed method, the classifiers can detect at least 12 buffer 


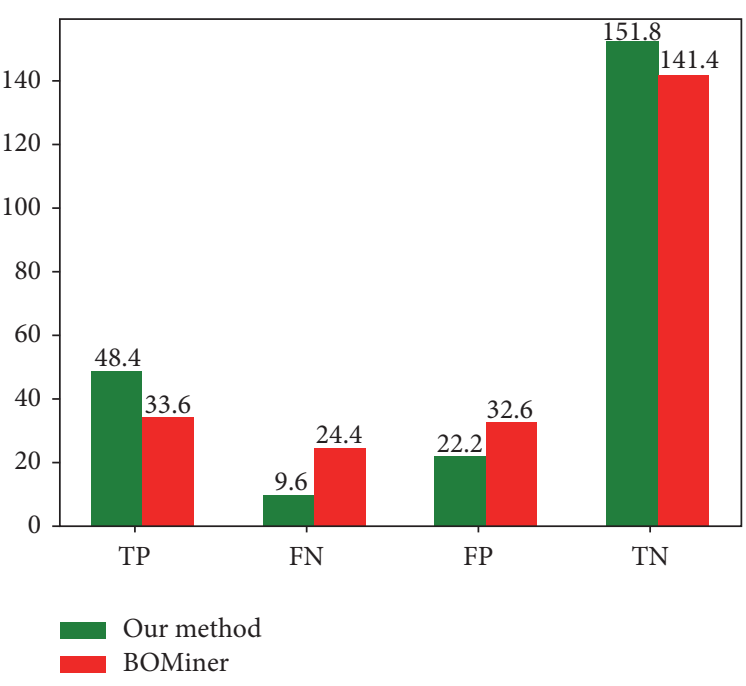

(a)

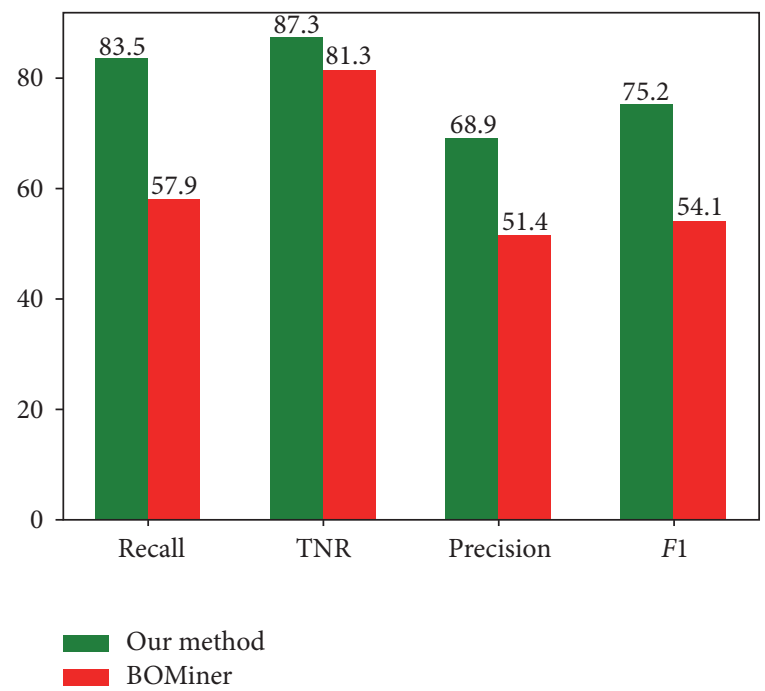

(b)

FIGURE 7: Comparison between our method and BOMiner. (a) Comparison of average confusion matrix. (b) Comparison of average recall, TNR, precision, and $F_{1}$.

TABLE 10: The comparison between Joern and our proposed method.

\begin{tabular}{|c|c|c|c|c|c|c|c|c|}
\hline & TP & FN & $\mathrm{FP}$ & $\mathrm{TN}$ & Recall (\%) & TNR (\%) & Precision (\%) & $F_{1}$ \\
\hline Joern & 6 & 12 & 2 & 177 & 33.3 & 98.9 & 75 & 46.2 \\
\hline $\mathrm{KNN}$ & 12 & 6 & 11 & 168 & 66.7 & 93.9 & 52.2 & 58.6 \\
\hline DT & 14 & 4 & 14 & 165 & 77.8 & 92.2 & 50 & 60.9 \\
\hline NB & 15 & 3 & 15 & 164 & 83.3 & 91.6 & 50 & 62.5 \\
\hline AdaBoost & 14 & 4 & 12 & 167 & 77.8 & 93.3 & 53.8 & 63.6 \\
\hline SVM & 16 & 2 & 17 & 162 & 88.9 & 90.5 & 48.5 & 62.8 \\
\hline
\end{tabular}

overflows and the least recall of the five classifiers is $66.7 \%$, which are all better than Joern. The highest TNR is $93.9 \%, 6 \%$ lower than Joern's $98.9 \%$. Figure 8 (a) shows the comparison of confusion matrix between our method and Joern. On average, our method detects 8.2 more TPs than Joern but outputs 11.8 more FPs. Figure 8 (b) shows the comparison of average recall, TNR, precision, and $F_{1}$. Our average recall is $78.9 \%, 45.6 \%$ higher than Joern and only 6.6\% lower at TNR. Our precision is $24.1 \%$ lower than Joern, while our $F_{1}$ is $15.5 \%$ higher than Joern. Two reasons can explain the low TP of Joern: (1) Joern only handles buffer overflows caused by dangerous function memcpy; (2) Joern employs two sanitization rules, dynamic allocation of the destination and relational expressions [4]; the rules fall into the region of our direct sanitization; the two rules would reduce false positives, while contributing to low TP.

5.3. Comparison to Flawfinder on Poppler 0.10.6. Poppler is a widely used open-source PDF library from which many buffer overflow vulnerabilities are detected. In this subsection, Poppler 0.10.6 is experimented on to evaluate our classifiers. As far as we are concerned, Poppler 0.10.6 has ten proven CVEs: CVE-2015-8868, CVE-2013-1788, CVE-20103704, CVE-2009-3938, CVE-2009-3608, CVE-2009-3607,
CVE-2009-3606, CVE-2009-3604, and CVE-2009-3603. We use these CVEs and our trained classifier to describe how our method assists in auditing buffer overflow vulnerabilities.

Poppler 0.10.6 source code is inputted and the output is described in Table 11. The TP, FN, FP, TN, recall, TNR, precision, and $F_{1}$ columns describe the performances of the five classifiers. We evaluate to what extent our method helps in code auditing based on the number of functions needed to be audited. The SVFs column contains the number of suspect vulnerable functions that need to be audited and the value of SVFs is the sum of TP and FP. Sink functions displays the number of functions that have buffers that satisfy one of the three sink types. All functions describes the total number of functions from the Poppler 0.10 .6 source code. The average TP is 8.8 , which means that we can find nearly 9 of 11 vulnerabilities. The reason why 9 CVEs have 11 vulnerabilities is that CVE-2013-1788 has 3 vulnerabilities. The average recall is $80 \%$ and the average TNR is $94 \%$. Because there are far more nonvulnerable functions than vulnerable functions, the precisions and $F_{1}$ are low where the average $F_{1}$ is $28.9 \%$ and the average precision is $17.7 \%$. Taking SVM as an example, code auditors only need to review 45 functions to find 10 of 11 vulnerabilities using our method. However, without our method, all 685 sink functions should be reviewed, which is a very heavy workload. 


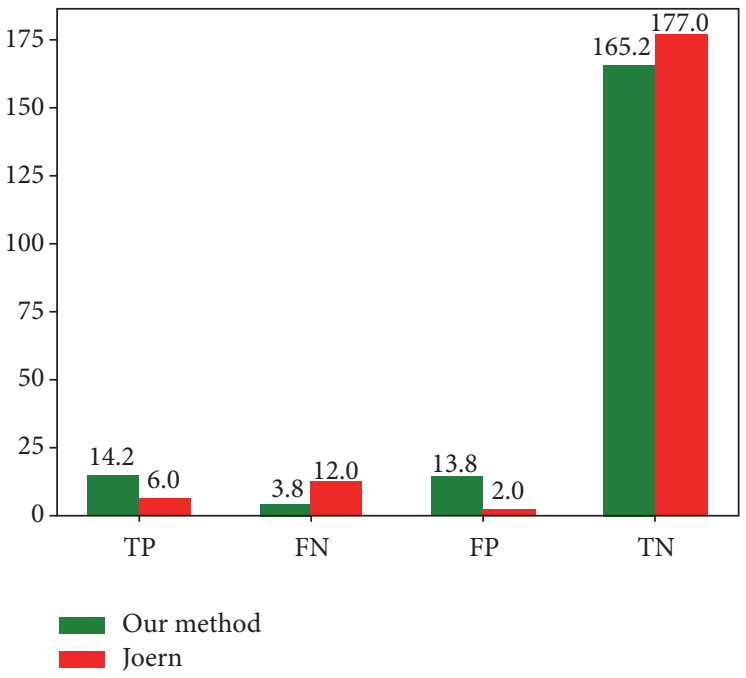

(a)

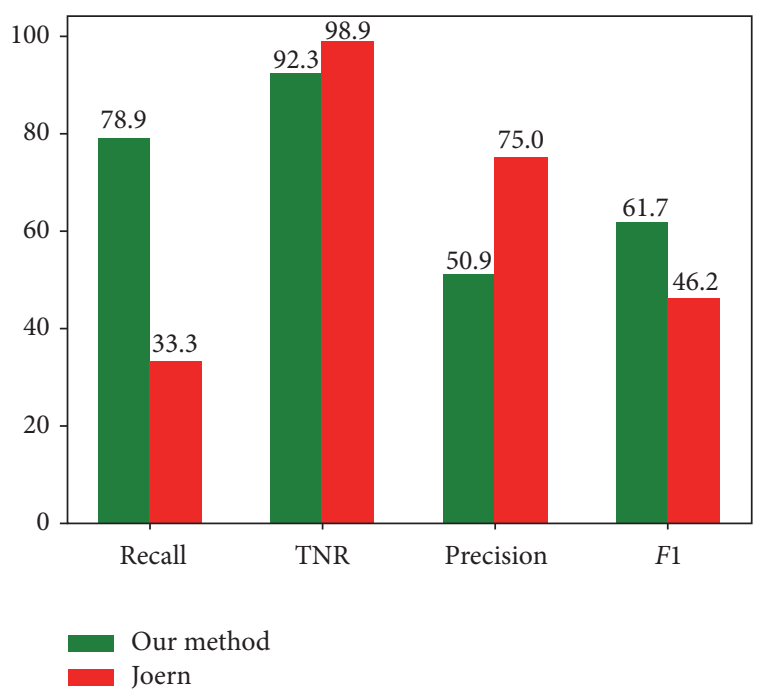

(b)

FIGURE 8: Comparison between our method and Joern. (a) Comparison of average confusion matrix. (b) Comparison of average recall, TNR, precision, and $F_{1}$.

TABLE 11: Performances of our five classifiers on Poppler 0.10.6.

\begin{tabular}{|c|c|c|c|c|c|c|c|c|c|c|c|}
\hline Classifier & $\mathrm{TP}$ & FN & FP & $\mathrm{TN}$ & Recall (\%) & TNR (\%) & Precision (\%) & $F_{1}(\%)$ & SVFs & Sink functions & All functions \\
\hline KNN & 7 & 4 & 37 & 648 & 63.6 & 94.6 & 15.9 & 25.4 & 44 & \multirow{5}{*}{685} & \multirow{5}{*}{4876} \\
\hline DT & 8 & 3 & 44 & 641 & 72.7 & 93.6 & 15.4 & 25.4 & 52 & & \\
\hline NB & 10 & 1 & 52 & 633 & 90.9 & 92.4 & 16.1 & 27.4 & 62 & & \\
\hline AdaBoost & 9 & 2 & 39 & 646 & 81.8 & 94.3 & 18.8 & 30.6 & 48 & & \\
\hline SVM & 10 & 1 & 35 & 650 & 90.9 & 94.9 & 22.2 & 35.7 & 45 & & \\
\hline
\end{tabular}

We also run Flawfinder 1.31 [14] on Poppler 0.10.6. Flawfinder is a static analyzer that scans for various kinds of vulnerabilities from source code. We identify all buffer overflow vulnerabilities from the output of Flawfinder. Flawfinder detected 8 of 11 vulnerabilities, which is slightly lower than the average performance of our method; however, it also generated 500 false positives, which is 12 times as many as ours. Thus, our method outperforms Flawfinder significantly in reducing the number of false positives of buffer overflow vulnerabilities, which definitely saves a lot of manual code auditing work.

\section{Related Work}

Static analysis tools fall into two categories: lightweight rough approaches and more thorough ones. Lightweight tools like Flawfinder [14] and Rats [15] are based on lexical analysis. Both translate source files to tokens and match them with certain vulnerable constructs in a library. Splint [16] can find abstract violations, unannounced modifications of global variables, and so forth, with manual annotations. For more thorough tools, Archer [17] symbolically computes buffer usage and employs a constraint solver to evaluate illegal memory accesses. Model checker [18] converts a buffer violation to a path to an error statement and then, using a constraint solver, verifies whether the path is feasible. Coventry
[19], Fortify [20], and CodeSonar [21] are commercial tools that require manual configuration work.

Dynamic test-case generation mainly involves two techniques, namely, fuzzing and symbolic execution. Fuzzing tools generate test cases to trigger program faults by mutating input bytes randomly. Recently, many methods [22-24] have been proposed to augment the fuzzing effect and [22] proved that a good seed test case contributed greatly to the fuzzing effect. Symbolic execution was first put forward by Clarke [25] and underwent great development because of the improvement of the constraint solver. Many tools were developed by various academic and research labs such as DART [26], CUTE [27], CREST [28], KLEE [29], SAGE [30], and S2E [31]. All of these tools fork a state once a branch instruction is encountered, which could lead to path explosion.

There are also many spot-on methods or tools that target buffer overflow vulnerability exclusively. Rawat and Mounier [32] implement an evolutionary computing approach to find buffer overflow, but it can only detect superficial faults. Another work from Rawat and Mounier [33] hunts buffer overflow in binary executables through a pattern obtained from "strcpy." Li et al. [34] utilized symbolic analysis representation to filter out irrelevant dependencies to scale to a large-scale code base for buffer overflow. Haller et al. [35] provided a guided fuzzing tool aimed only at array boundary violations. 
Recently, machine learning algorithms have been applied in the vulnerability detection field. Yamaguchi et al. [6] proposed a vulnerability extrapolation method to assist code auditors, using the similarity in AST structure of similar functions. The effectiveness of this method depends on the existence of a similar function of a known vulnerability. Yamaguchi et al. also leveraged anomaly detection to identify missing checks of buffers [36] and applied a clustering algorithm to taint-style vulnerabilities [37]. Padmanabhuni and Tan's work [38] is the closest to our work, but it did not provide the concept of complexity, which is very important in modern software.

\section{Conclusion}

In this paper, a method that assists in auditing buffer overflow vulnerabilities using machine learning is proposed. We define seven kinds of static code attributes according to the 22 taxonomies of buffer overflow vulnerabilities and also design the extended code property graph to extract these attributes. Then the digitalized attributes are used to train five classifiers. In our experiment, the classifiers reached an average recall of $83.5 \%$, average true negative rate of $85.9 \%$, a best recall of $96.6 \%$, and a best true negative rate of $91.4 \%$. Due to the imbalance of the training samples, the average precision of the classifiers is $68.9 \%$ and the average $F_{1}$ score is $75.2 \%$. We then applied the classifiers to a real program, Poppler 0.10 .6 . Our classifiers outperformed Flawfinder significantly by reducing the false positive rate to $1 / 12$. In conclusion, our method can assist in auditing buffer overflow vulnerabilities in source code.

\section{Conflicts of Interest}

The authors declare that there are no conflicts of interest regarding the publication of this paper.

\section{References}

[1] W. Le and M. L. Soffa, "Generating analyses for detecting faults in path segments," in Proceedings of the 20th International Symposium on Software Testing and Analysis, ISSTA 2011, pp. 320-330, Canada, July 2011.

[2] C. Cadar and K. Sen, "Symbolic execution for software testing: Three decades later," Communications of the ACM, vol. 56, no. 2, pp. 82-90, 2013.

[3] K. J. Kratkiewicz, Evaluating Static Analysis Tools for Detecting Buffer Overflows in C Code, Harvard University, Boston, CA, USA, 2005.

[4] F. Yamaguchi, N. Golde, D. Arp, and K. Rieck, "Modeling and discovering vulnerabilities with code property graphs," in Proceedings of the 35th IEEE Symposium on Security and Privacy, SP 2014, pp. 590-604, San Jose, CA, USA, May 2014.

[5] L. Moonen, "Generating robust parsers using island grammars," in Proceedings of the Eighth Working Conference on Reverse Engineering, Stuttgart, Germany, 2-6 October, 2001.

[6] F. Yamaguchi, M. Lottmann, and K. Rieck, "Generalized vulnerability extrapolation using abstract syntax trees," in Proceedings of the 28th Annual Computer Security Applications Conference
(ACSAC '12), pp. 359-368, ACM, Orlando, Fla, USA, December 2012.

[7] CVE-2016-9537, https://cve.mitre.org/cgi-bin/cvename.cgi? name $=$ CVE-2016-9537.

[8] libtiff-4.0.6, https:/github.com/vadz/libtiff/releases/tag/Releasev4-0-6.

[9] M. Zitser, R. Lippmann, and T. Leek, "Testing static analysis tools using exploitable buffer overflows from open source code," in Proceedings of the the 12th ACM SIGSOFT twelfth international symposium, p. 97, Newport Beach, CA, USA, October 2004.

[10] M. A. Rodriguez and P. Neubauer, "The Graph Traversal Pattern," in Graph Data Management: Techniques and Applications, S. Sakr and E. Pardede, Eds., pp. 29-46, IGI Global, Hershey, PA, USA, 1st edition, 2012.

[11] scikit-learn, http://scikit-learn.org/stable/.

[12] S. V. Stehman, "Selecting and interpreting measures of thematic classification accuracy," Remote Sensing of Environment, vol. 62, no. 1, pp. 77-89, 1997.

[13] B. M. Padmanabhuni and H. B. K. Tan, "Predicting buffer overflow vulnerabilities through mining light-weight static code attributes," in Proceedings of the 25th IEEE International Symposium on Software Reliability Engineering Workshops, ISSREW 2014, pp. 317-322, Naples, Italy, November 2014.

[14] Flawfinder, https://www.dwheeler.com/flawfinder/.

[15] Rats, https://code.google.com/archive/p/rough-auditing-toolfor-security/.

[16] Splint, http://splint.org.

[17] Y. Xie, A. Chou, and D. Engler, "ARCHER: Using Symbolic, Path Sensitive Analysis to Detect Memory Access Errors," in In Proceedings of 11th ACM SIGSOFT International Symposium on Foundations of Software Engineering, Helsinki, Finland, September 2003.

[18] L. Wang, Q. Zhang, and P. Zhao, "Automated detection of code vulnerabilities based on program analysis and model checking," in Proceedings of the 8th IEEE International Working Conference on Source Code Analysis and Manipulation, SCAM 2008, pp. 165-173, Beijing, China, September 2008.

[19] Coverity, https://scan.coverity.com/.

[20] Fortify, http://www.fortify.net/.

[21] CodeSonar, https://www.grammatech.com/products/codesonar.

[22] A. Rebert, S. K. Cha, T. Avgerinos et al., "Optimizing Seed Selection for Fuzzing," in In the Proceedings of the 23rd USENIX Security Symposium, San Diego, CA, USA, August 2014.

[23] M. Woo, S. K. Cha, S. Gottlieb, and D. Brumley, "Scheduling Black-box Mutational Fuzzing," in In Proceedings of the 2013 ACM SIGSAC Conference on Computer and Communications Security, New York, NY, USA, November 2013.

[24] W. Wang, H. Sun, and Q. Zeng, "SeededFuzz: Selecting and Generating Seeds for Directed Fuzzing," in Proceedings of the 10th International Symposium on Theoretical Aspects of Software Engineering, TASE 2016, Shanghai, China, July 2016.

[25] L. A. Clarke, "A program testing system," in In Proceedings of the 1976 Annual Conference, pp. 488-491, Houston, Texas, United States, October 1976.

[26] P. Godefroid, N. Klarlund, and K. Sen, "DART: Directed Automated Random Testing," in In Proceedings of the 2005 ACM SIGPLAN Conference on Programming Language Design and Implementation, PLDI 05, pp. 213-223, Chicago, IL, USA, June 2005. 
[27] K. Sen and G. Agha, "CUTE and jCUTE : Concolic Unit Testing and Explicit Path Model-Checking Tools," in In Proceedings of the 18th International Conference on Computer-Aided Verificatio, Seattle, WA, USA, August 2006.

[28] J. Burnim and K. Sen, "Heuristics for scalable dynamic test generation," in Proceedings of the ASE 2008 - 23rd IEEE/ACM International Conference on Automated Software Engineering, pp. 443-446, L’Aquila, Italy, September 2008.

[29] C. Cadar, D. Dunbar, and D. Engler, "KLEE: Unassisted and automatic generation of high-coverage tests for complex systems programs," in OSDI'08 Proceedings of the 8th USENIX conference on Operating systems design and implementation, San Diego, Calif, USA, December 2008.

[30] B. Elkarablieh, P. Godefroid, and M. Y. Levin, "Precise pointer reasoning for dynamic test generation," in Proceedings of the 18th International Symposium on Software Testing and Analysis, ISSTA 2009, Chicago, IL, USA, July 2009.

[31] V. Chipounov, V. Kuznetsov, and G. Candea, "S2E: A platform for in-vivo multi-path analysis of software systems," in Proceedings of the 16th International Conference on Architectural Support for Programming Languages and Operating Systems, ASPLOS 2011, pp. 265-278, Newport Beach, CA, USA, March 2011.

[32] S. Rawat and L. Mounier, "An evolutionary computing approach for hunting buffer overflow vulnerabilities: A case of aiming in dim light," in Proceedings of the 6th European Conference on Computer Network Defense, EC2ND 2010, pp. 37-45, Berlin, Germany, October 2010.

[33] S. Rawat and L. Mounier, "Finding buffer overflow inducing loops in binary executables," in Proceedings of the 2012 IEEE 6th International Conference on Software Security and Reliability, SERE 2012, pp. 177-186, Waikiki, HONO, USA, June 2012.

[34] L. Li, C. Cifuentes, and N. Keynes, "Practical and effective symbolic analysis for buffer overflow detection," in Proceedings of the 18th ACM SIGSOFT International Symposium on the Foundations of Software Engineering, FSE-18, pp. 317-326, Santa Fe, NM, USA, November 2010.

[35] I. Haller, A. Slowinska, M. Neugschwandtner, and H. Bos, "Dowsing for Overflows: A Guided Fuzzer to Find Buffer Boundary Violations," in In Proceedings of the 22nd USENIX Security Symposium, Washington, DC, USA, August 2013.

[36] F. Yamaguchi, C. Wressnegger, and H. Gascon, "Chucky: Exposing missing checks in source code for vulnerability discovery," in Proceedings of the 2013 ACM SIGSAC Conference on Computer and Communications Security, CCS 2013, pp. 499-510, Berlin, Germany, November 2013.

[37] F. Yamaguchi, A. Maier, H. Gascon, and K. Rieck, "Automatic inference of search patterns for taint-style vulnerabilities," in Proceedings of the 36th IEEE Symposium on Security and Privacy, SP 2015, pp. 797-812, San Jose, CA, USA, May 2015.

[38] B. M. Padmanabhuni and H. B. K. Tan, "Auditing buffer overflow vulnerabilities using hybrid static-dynamic analysis," in Proceedings of the 38th Annual IEEE Computer Software and Applications Conference, COMPSAC 2014, pp. 394-399, Vasteras, Sweden, July 2014. 


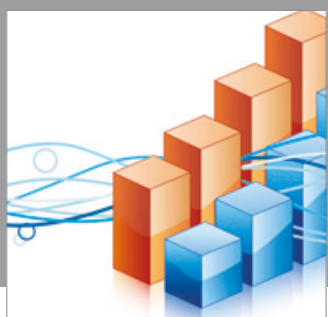

Advances in

Operations Research

vatersals

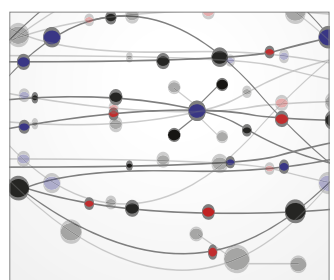

\section{The Scientific} World Journal
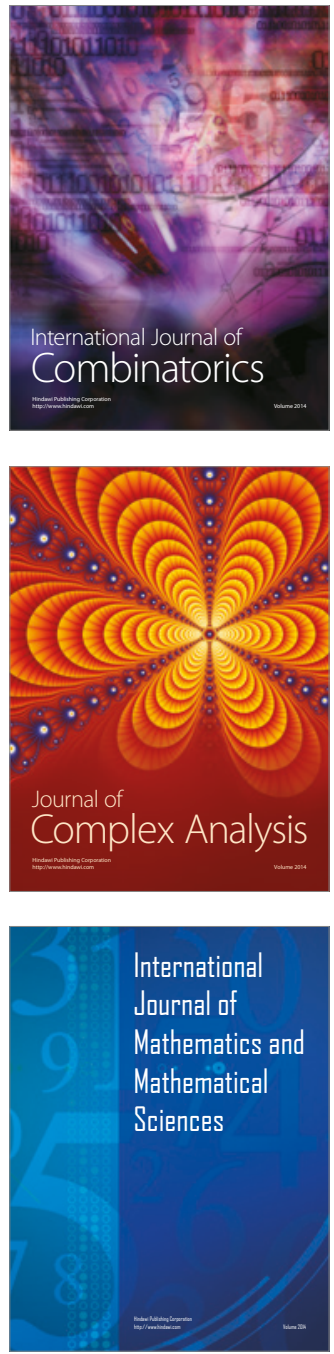
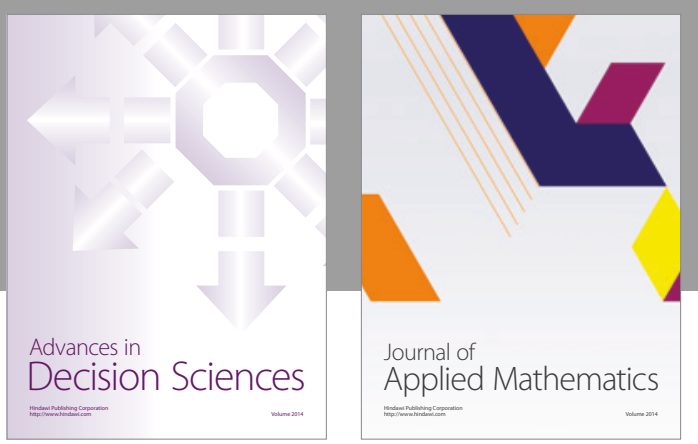

Algebra

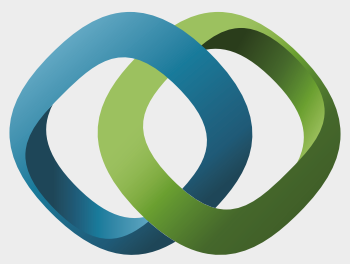

\section{Hindawi}

Submit your manuscripts at

https://www.hindawi.com
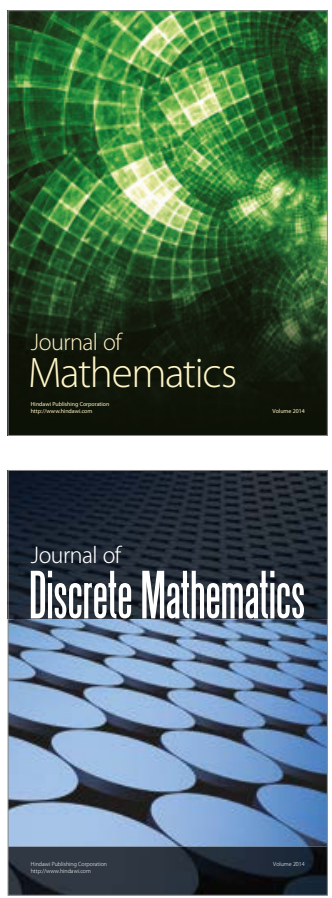

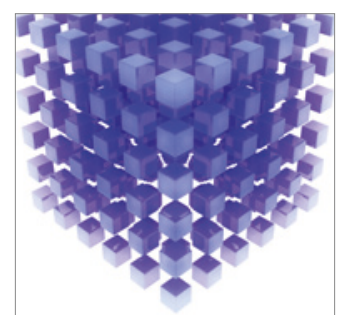

Mathematical Problems in Engineering
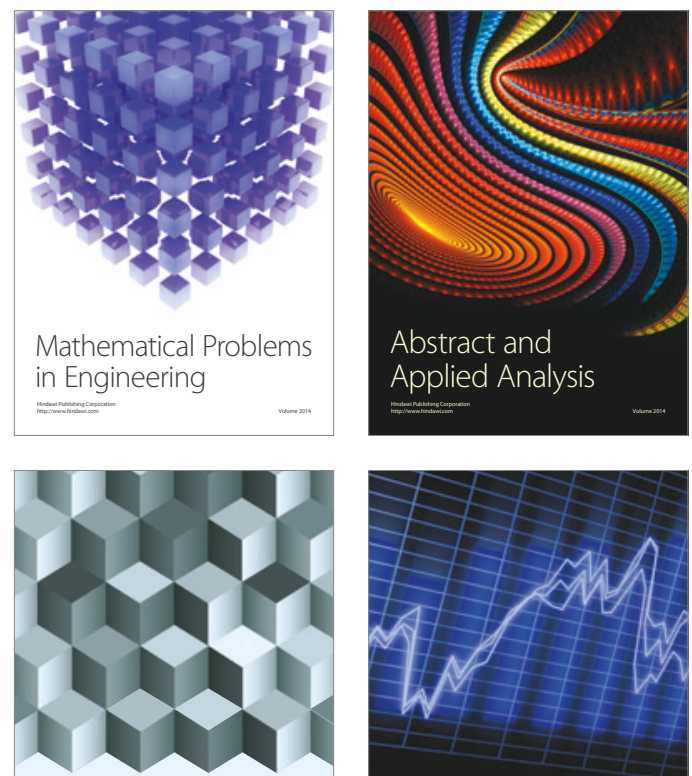

Journal of

Function Spaces

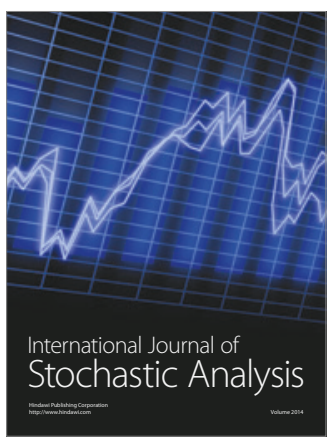

Probability and Statistics
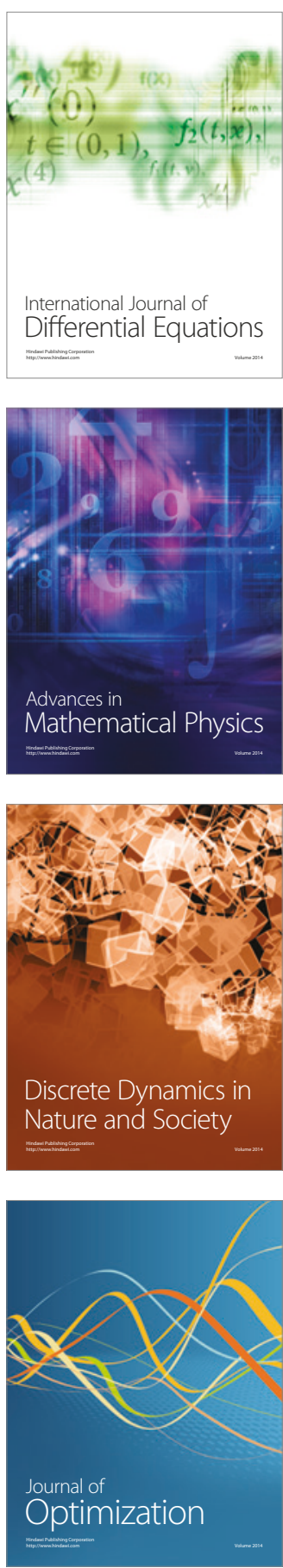